\title{
Les politiques publiques menées sur les chemins de Saint- Jacques-de-Compostelle et leur perception par les marcheurs- pèlerins du XXle siècle
}

\author{
Christophe Alcantara \\ Université Toulouse 1 Capitole, France
}

\begin{abstract}
This article proposes to analyze the nature of the public policies carried out in the management and the development of the roads of Saint-Jacques-de-Compostelle since about thirty years, as well as the incidence of these actions in the representation of the paths of Saint -Jacques-de-Compostelle by the pilgrim walkers. After reviewing some facts and figures relating to the paths, this artist reflects on the social and economic forces that are empowered by these paths. Finally, this article addresses the importance of cultural paths in the production of local development strategies.
\end{abstract}

Keywords: pilgrims, cultural heritage, paths of Santiago de Compostela, public policy

Résumé : Cet article propose d'analyser la nature des politiques publiques menées dans la gestion et le développement des chemins de Saint-Jacques-de-Compostelle depuis une trentaine d'années, ainsi que l'incidence de ces actions dans la représentation des chemins de Saint-Jacques-de-Compostelle par les marcheurs-pèlerins. Après avoir fait un retour sur quelques faits et chiffres relatifs aux chemins, cet artiste porte une réflexion sur les forces sociales et économiques qui sont habilitées par ces chemins. Enfin, cet article aborde l'importance des chemins culturels dans la production de stratégies de développement local.

Mots clés : pèlerins, patrimoine, chemins de Saint-Jacques-de-Compostelle, politiques publiques

\section{Introduction}

Cet article propose d'analyser la nature des politiques publiques menées dans la gestion et le développement des chemins de Saint-Jacques-de-Compostelle depuis une trentaine d'années, ainsi que l'incidence de ces actions dans la représentation des chemins de Saint-Jacques-deCompostelle par les marcheurs-pèlerins. La méthodologie repose sur des entretiens semidirectifs menés auprès de décideurs locaux en charge de la valorisation des chemins ainsi que des entretiens approfondis, reposant sur une démarche de sociologie compréhensive, réalisés auprès de marcheurs-pèlerins ayant accompli tout ou partie du chemin en direction de SaintJacques-de-Compostelle. Elle est également complétée par l'analyse des comptes Instagram de marcheurs pèlerins avec une qualification de près de 4000 photos publiées sur ce réseau social. Cet article abordera successivement le renouveau de la fréquentation des chemins de SaintJacques-de-Compostelle, puis l'analyse des politiques publiques à l'œuvre sur les chemins de

Christophe Alcantara est enseignant-chercheur en Sciences de l'Information et de la Communication à Université Toulouse 1 Capitole. Courriel : christophe.alcantara@ut-capitole.fr 
Saint-Jacques-de-Compostelle et enfin la perception et représentation des chemins par les marcheurs-pèlerins en route vers Saint-Jacques-de-Compostelle.

\section{Les chemins de Saint-Jacques-de-Compostelle : un phénomène de société et une géographie renouvelée}

Un pèlerinage est une visite, un déplacement vers un sanctuaire. Cette définition générique est commune à la plupart des religions. Le grand pèlerinage à la Mecque que chaque musulman doit accomplir au moins une fois dans sa vie est l'un des cinq piliers de l'Islam. De la même façon, des pèlerinages caractérisent de façon spécifique l'hindouisme, le bouddhisme et le christianisme. Les trois principaux pèlerinages du monde chrétien sont ceux qui mènent à Jérusalem, à Rome et à Saint-Jacques-de-Compostelle. À Jérusalem, le pèlerin veut se rapprocher du Saint Sépulcre et de la Passion du Christ ; à Rome, il souhaite s'inscrire dans les pas de Pierre, apôtre et père de l'Église ; à Saint-Jacques-de-Compostelle, on se recueille auprès du sépulcre de Saint-Jacques le Majeur, fils de Zébédée, frère de Saint-Jean l'Évangéliste, l'un des trois apôtres présents le jour de la transfiguration du Christ. Jacques dit le Majeur fut également le premier des douze apôtres à subir le martyre. Chaque apôtre avait reçu une partie du monde à évangéliser, et Jacques le Majeur devait assurer sa mission dans la péninsule ibérique. L'histoire de la translation miraculeuse du corps de l'apôtre au lendemain de son martyre est le fondement à partir duquel on a inventé l'avènement du tombeau de l'apôtre aux alentours de 830. L'engouement devient alors considérable pour un ensemble de pèlerins. Au Moyen-Âge, le pèlerinage était un horizon très structurant des relations sociales et de l'imaginaire des populations. La charge spirituelle était considérable et la symbolique du pèlerinage de Saint-Jacques l'était tout autant. "L'existence d'enseignes spécifiques, les coquilles du pecten maximus, dotèrent le sanctuaire apostolique d'un capital symbolique incomparable » (Rucquoi, 2014, p. 11). L'expression " pèlerin » qui qualifiait initialement ceux qui marchaient exclusivement sur les routes de Compostelle est devenue le terme consacré pour qualifier l'ensemble des croyants qui réalisent un pèlerinage vers un sanctuaire. Étymologiquement, le mot pèlerinage viendrait des expressions latines peragrere, c'est-à-dire aller au loin, ou de peregrinus, l'étranger. Historiquement « le pèlerinage relève avant tout de la religion » (Rucquoi, 2014, p. 13).

\section{Quelques chiffres sur ce phénomène de société}

Les chemins de Saint-Jacques-de-Compostelle deviennent un véritable phénomène de société. En effet, la fréquentation des chemins n'a cessé de croître depuis la fin des années 1990. On est passé d'une fréquentation moyenne de 25000 pèlerins en 1997 à plus de 154000 en 1999. La croissance régulière de cet engouement s'est confirmée tout au long des 20 dernières années pour atteindre le nombre historique de 327378 pèlerins arrivés et recensés en 2018 par le bureau des pèlerins de la cathédrale de Saint-Jacques-de-Compostelle ${ }^{1}$ contre 301036 en 2017. Cette croissance continue de la fréquentation des chemins s'opère alors même que l'on constate un effondrement de la pratique religieuse chrétienne en Europe et plus particulièrement en France. 


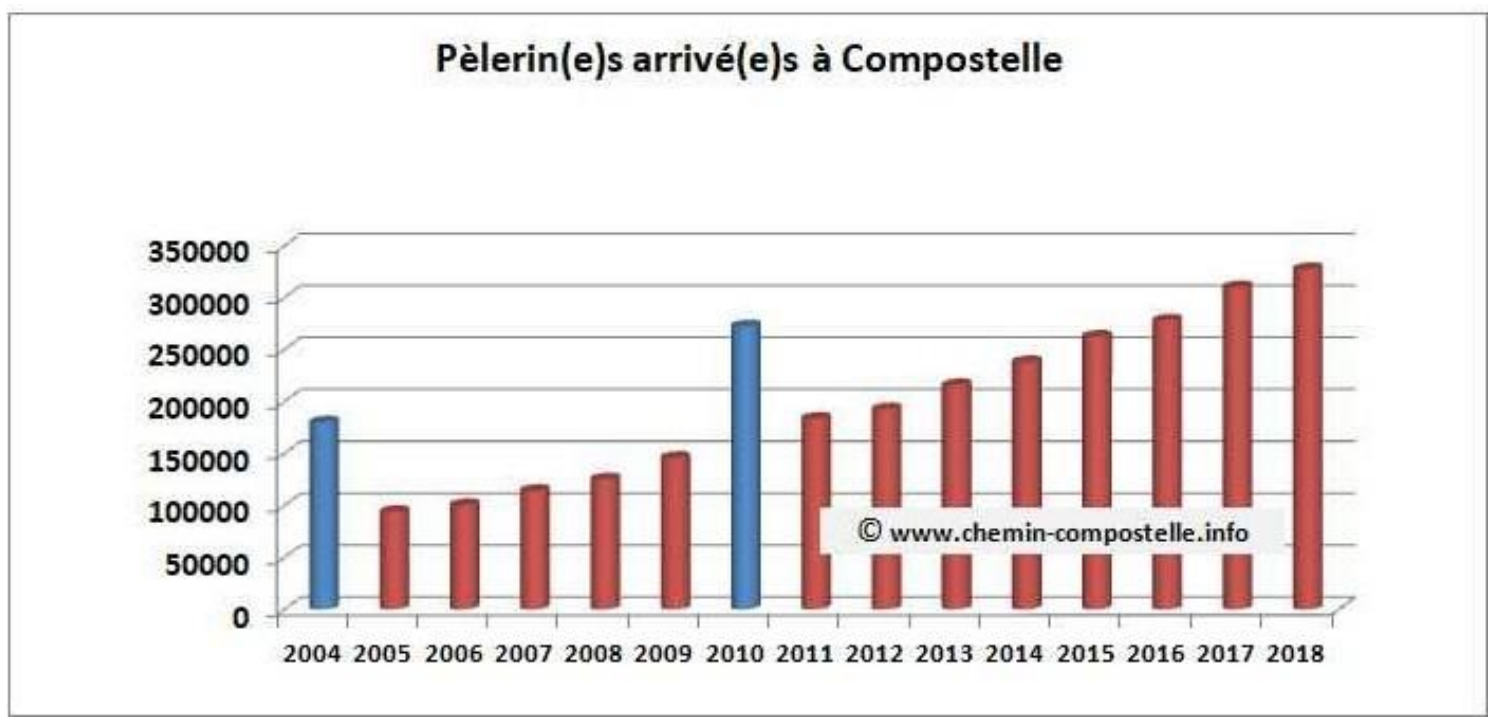

Figure 1 : Nombre de pèlerins arrivés à Compostelle de 2004 à 2018

En référence au tableau joint, les années 2004 et 2010 sont des années jacquaires, c'est-àdire des années où la Saint-Jacques est un dimanche ce qui génère alors un pic annuel de fréquentation. Pour compléter les données sur la fréquentation, on peut noter qu'il y a $50 \%$ de femmes et $50 \%$ d'hommes. L'ordre d'importance par nationalité ne change presque pas par rapport à 2017, avec 44\% d'Espagnols (soit 144141 pèlerins). Sur le nombre total d'étrangers (183 237), les principaux contingents sont italiens $(14,7 \%)$, allemands $(13,81 \%)$, américains $(10,14 \%)$, portugais $(7,8 \%)$ et français $(4,78 \%)$.

\section{La géographie des chemins de Saint-Jacques-de-Compostelle}

La route exprime une relation. Elle est un passage, le lien entre les lieux. Elle est le support, une construction, une histoire produite par les hommes. Par essence, la route est ouverte. Elle appartient à tous puisque chacun peut l'emprunter. " La route a progressivement remplacé la voie, plus civilisée ou mieux viabilisée [...]. L'état des routes trace le chemin des conquérants » (Vallet, 1996, p. 293). Le chemin quant à lui, renvoie à un imaginaire passé, où l'on prend le temps de voir et d'observer. On est alors sur une autre échelle d'espace-temps, tout à fait anachronique avec la période actuelle.

Au Moyen-Âge, faut-il le rappeler, les chemins de Saint-Jacques-de-Compostelle n'existaient pas de façon formelle. Le pèlerin quittait sa maison en direction de Compostelle à pied ou à cheval et il utilisait les voies de communication à sa disposition. Il se mêlait aux autres voyageurs sur les routes qui n'avaient pas pour objectif initial de mener à Compostelle. Sa seule contrainte était logistique : il devait être à une distance accessible d'un sanctuaire ou d'un lieu d'accueil destiné aux pèlerins. "Le chemin de Saint-Jacques est une dénomination de mémoire qui, avant le renouveau tout récent du pèlerinage, attestait dans la tradition orale et dans la toponymie le souvenir d'un passage de nombreux pèlerins sur tel ou tel tronçon " (Julia, 2016, p. 148). C'est donc avant tout une mémoire collective constituée des traces et témoignages produits au cours du dernier millénaire. Les contours sont ainsi poreux et peuvent se déplacer 
au fil des marqueurs visibles sur les chemins. II n'en demeure pas moins qu'une source historique fait référence et produit la matrice générique du chemin espagnol et des quatre chemins français, c'est le Codex Calixtinus. Compilation d'un ensemble de textes qui remontent pour les premiers au XIIème siècle, son écriture s'étale sur deux ou trois siècles. Le Vème livre du Codex Calixtinus actuellement connu sous le nom de " guide du pèlerin de Saint-Jacques-deCompostelle " (Vielliard, 1938) décrit longuement l'itinéraire en Espagne et traite des grandes lignes des quatre chemins français, à savoir les chemins qui viennent de Tours, du Vézelay, du Puy en Velay et d'Arles. Ces quatre chemins convergent tous à Punta de la Reina en Espagne et le chemin principal qui conduit alors à Saint-Jacques-de-Compostelle s'appelle Camino Frances.

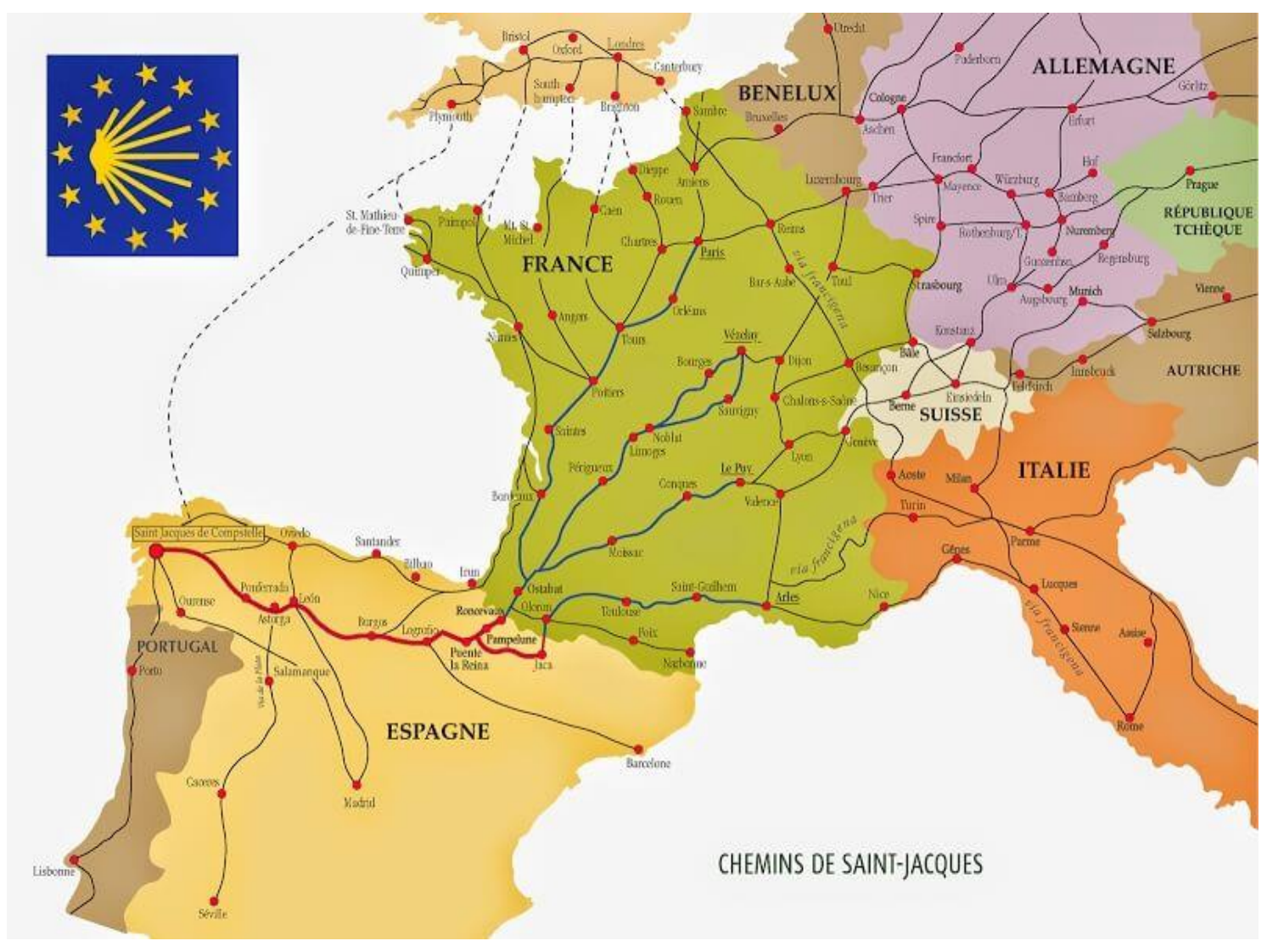

Figure 2 : Les quatre principaux itinéraires français et le Camino Frances

À partir de cette géographie construite par les hommes, il est intéressant à présent de voir comment des politiques publiques se sont structurées au fil du temps pour développer et pérenniser les chemins de Saint-Jacques-de-Compostelle.

\section{Les politiques publiques mises en œuvre pour promouvoir et développer les chemins de Saint-Jacques-de-Compostelle}

Historiquement, ce sont les souverains qui vont promouvoir les chemins et les aménager pour assurer la sécurité et l'accueil des pèlerins. Le roi des Asturies Alphonse III réalise ainsi la construction du sanctuaire en 899 . A partir de 1070, les différents souverains créent un réseau 
d'accueil dans les villes et villages avec des haltes tous les trente kilomètres pour permettre l'essor et l'accueil des pèlerins. II y a également en parallèle un enjeu politique qui est de valoriser l'image de Santiago de Compostella, et son incarnation iconographique Santiago Matamoros, figure mythique de la Reconquista espagnole. En 1257, les infrastructures qui bordent les chemins se renforcent avec la création des hôpitaux publics, dont l'Hôtel-Dieu de Toulouse (voir figure 3 ) est une construction emblématique. Ce monument est d'ailleurs inscrit au patrimoine mondial de l'UNESCO.

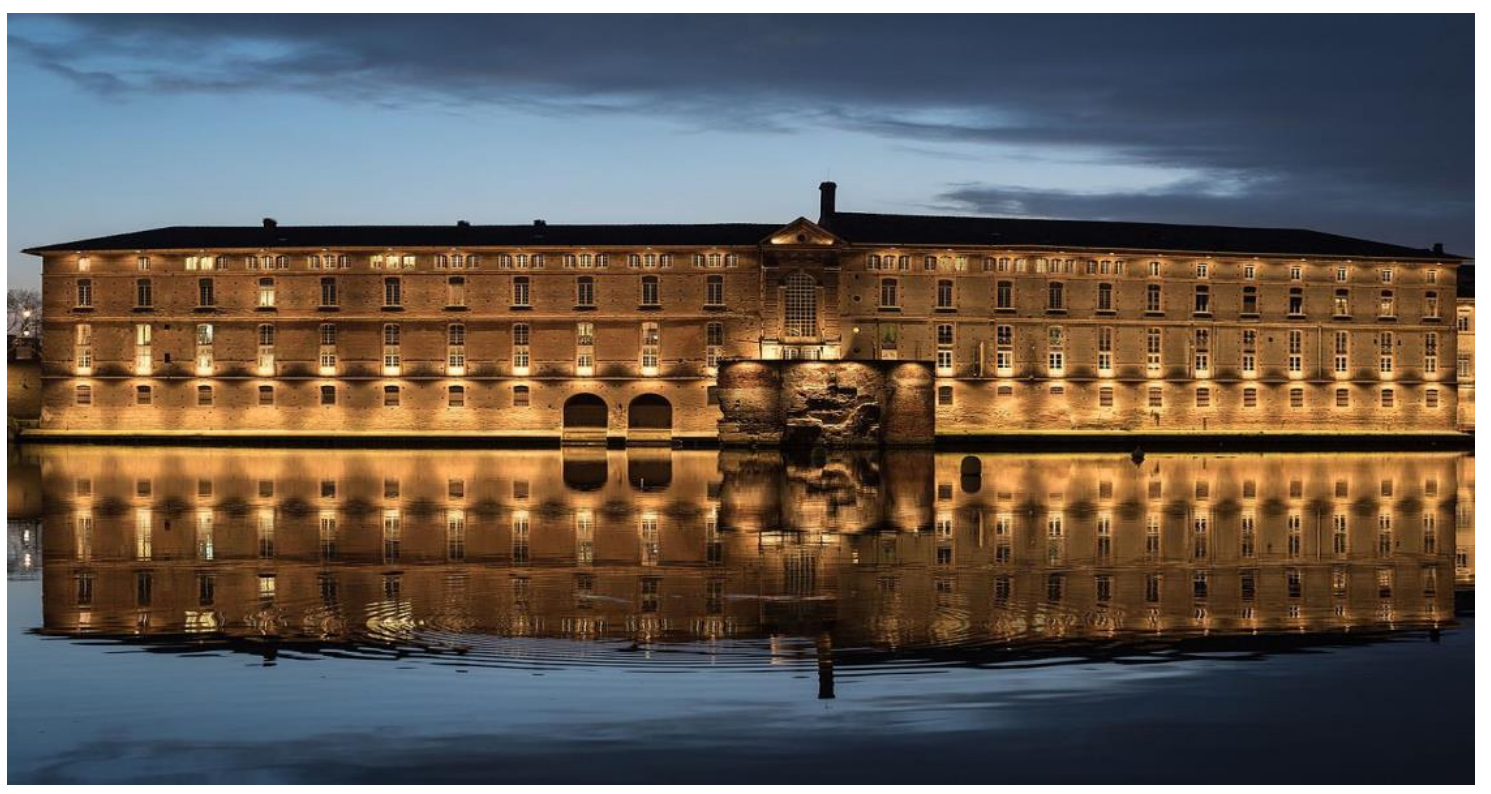

Figure 3 : L'Hôtel-Dieu de Toulouse, l'un des 64 monuments du bien 868, inscrit au patrimoine mondial de l'UNESCO, dit « chemins de Saint-Jacques-deCompostelle en France »

Beaucoup plus près de nous, 1982, année jacquaire, marque la première fois où un pape se rend à Compostelle. II s'agit de Jean-Paul II, pape très politique qui profite de ce déplacement pour rappeler les racines chrétiennes de l'Europe. En 1987, les chemins de Saint-Jacques-deCompostelle inaugurent le programme des itinéraires culturels européens du Conseil de l'Europe. Ce label a pour objectif de participer à la construction européenne en s'appuyant sur le patrimoine et en favorisant la valorisation de la mémoire, de l'histoire et du patrimoine européen. Il est donc assez remarquable de constater que le Conseil de l'Europe inaugure le premier itinéraire culturel européen par une appropriation politique du pèlerinage historique vers Saint-Jacques-de-Compostelle. Ce fait historique devient alors un marqueur de l'identité européenne en cours de construction. La figure du pèlerin qui se déplace à travers les régions, les pays et les frontières pour accéder au sanctuaire au bout de la Galice devient l'incarnation moderne du projet politique de construction européenne avec ses corollaires que sont l'espace Schengen, la monnaie unique et le mythe d'une identité européenne. Ce qui, pendant des siècles, était un phénomène religieux, est devenu, grâce à la déclaration du Conseil de l'Europe en 1987 et à la mise en place d'une signalétique européenne commune (figure 4), un itinérairesymbole, vivant, un marqueur dynamique de la construction d'une identité européenne. 


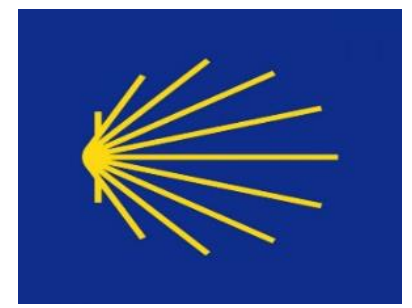

Figure 4 : Coquille Saint-Jacques stylisée jaune sur fond bleu, symbole du label « itinéraire culturel du Conseil de l'Europe » attribué aux chemins de SaintJacques-de-Compostelle

\section{Inscription des chemins de Saint-Jacques-de-Compostelle au patrimoine mondial de I'UNESCO}

Il est important de préciser que les critères principaux de sélection d'un bien par l'UNESCO sont : la valeur universelle exceptionnelle, l'authenticité, l'intégrité et enfin l'existence de garanties pour la conservation et la gestion du bien. L'inscription des chemins de Saint-Jacques-deCompostelle a été réalisée en deux temps. En 1993, I'UNESCO a inscrit le Camino Frances en Espagne, soit 700 kilomètres de sentiers de Punta de la Reina à Santiago. En 1998, I'UNESCO inscrit le bien culturel " chemins de Saint-Jacques-de-Compostelle en France ». Ce bien, appelé très souvent par son numéro d'inscription, à savoir le bien 868 , est tout à fait singulier car c'est la première fois que I'UNESCO inscrit un ensemble de 78 éléments, constitué de 64 monuments, 7 sections de sentiers et 7 ensembles (figure 5). C'est donc un bien particulier, par sa nature polymorphe et hétérogène, relevant à fois du patrimoine matériel et immatériel inscrit au patrimoine mondial et couvrant les quatre voies historiques des chemins de Saint-Jacques qui traversent la France. Cet objet nouveau, constitué par l'inscription des chemins de Saint-Jacques au patrimoine mondial de I'UNESCO, a également permis de déplacer la perception politique de la nature des chemins. Il y a eu un glissement d'un bien religieux vers un bien culturel. Cela est le fruit de la double labellisation successive du Conseil de l'Europe et de l'UNESCO. 


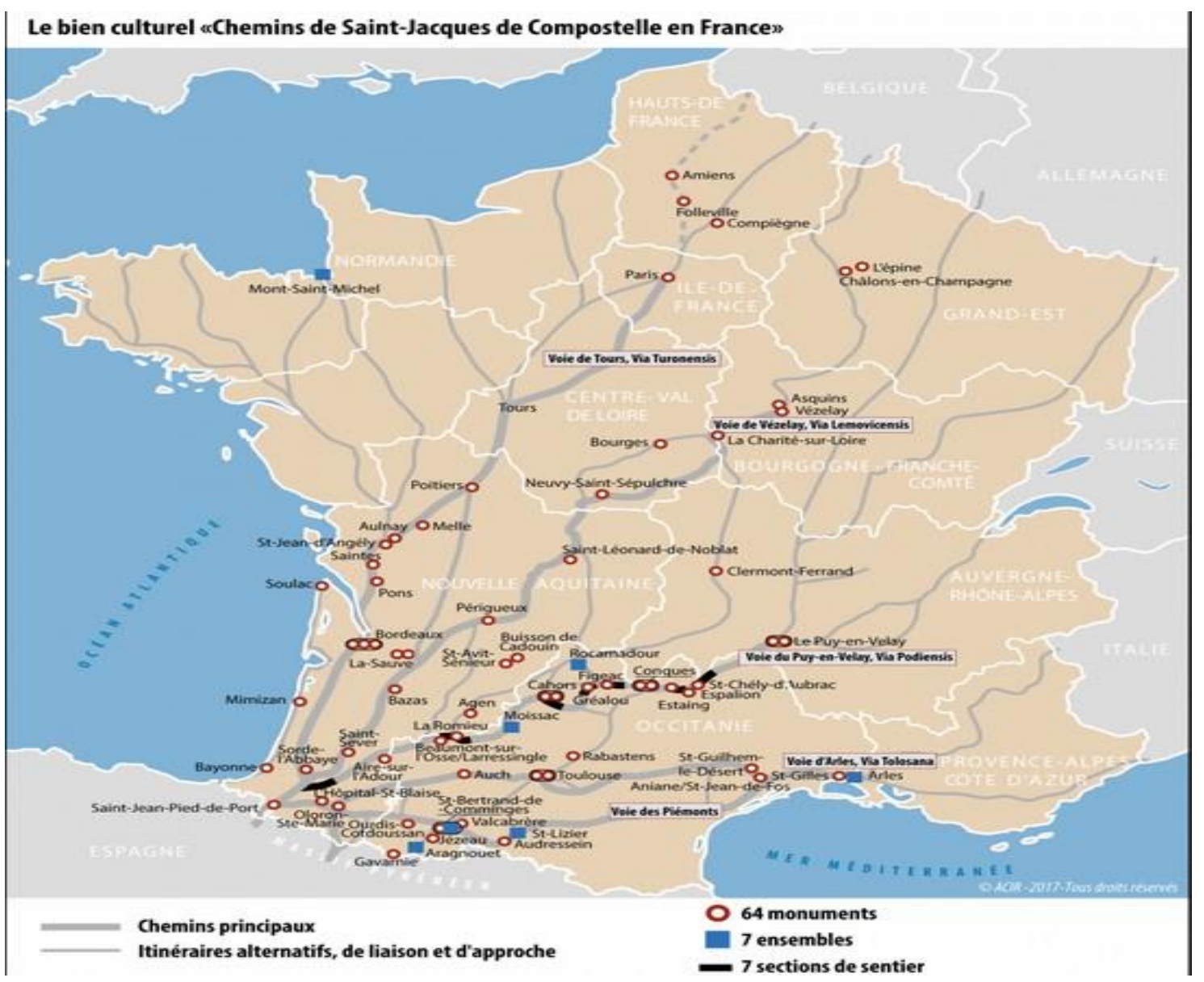

Figure 5 : Le bien 868 inscrit au patrimoine mondial de I'UNESCO

\section{Nature des politiques régionales menées sur les chemins de Saint- Jacques-de-Compostelle}

Notre propos est forcément réducteur car il concerne exclusivement l'analyse de la politique menée par la région Occitanie qui a la particularité d'intégrer deux des chemins historiques, à savoir le chemin du Puy-en-Velay et le chemin d'Arles. Cette région s'étend jusqu'à la frontière espagnole où l'ensemble des 4 chemins du Codex Calixtinus convergent pour ensuite devenir un unique chemin, le Camino Frances. Il faut d'emblée remarquer que l'inscription des chemins au patrimoine mondial de l'UNESCO a fait basculer les chemins de la sphère religieuse vers le champ de la culture. Ce label n'est pas la cause unique de ce basculement. En effet, la pratique religieuse chrétienne, et plus particulièrement catholique, est en perte de vitesse considérable. L'Église est donc de plus en plus marginalisée face à la mainmise des pouvoirs publics pour administrer les chemins. On constate un changement de destination, un glissement du but vers le moyen. En effet, la destination Saint-Jacques-de-Compostelle est historiquement le but initial de la marche des pèlerins et les politiques menées au cours de l'histoire servaient dans une large partie à servir, accompagner, réguler les flux de pèlerins jusqu'à la destination finale. Aujourd'hui, les pouvoirs publics concentrent leur politique sur les moyens, à savoir les chemins qui deviennent alors un but en soi. C'est en ce sens qu'il y a une appropriation civile des chemins 
de Saint-Jacques-de-Compostelle qui assure une valorisation du moyen au détriment du but. Pour certains décideurs régionaux, le bien 868 est devenu un produit culturel, un levier de croissance économique, qui plus est dans des zones rurales où les moteurs de croissance sont peu nombreux. À ce sujet, l'intervention le 26 octobre 2018 de l'ancien président de la région Occitanie, Martin Malvy (2018) est assez significative. Selon lui,

en 2017, il y avait 1,225 milliard de touristes dans le monde, il y en aura 2 milliards à l'horizon 2025. Le patrimoine c'est la motivation d'un visiteur sur deux en France [...]. II faut accroitre la fréquentation des chemins de SaintJacques car le tourisme de masse n'est pas acté sur ce patrimoine, 300000 pèlerins par an, c'est peu.

À partir d'une telle déclaration, on voit bien toute la volonté d'instrumentaliser les chemins et de travestir leur nature historique pour en faire un autre objet, d'une autre nature. On peut comprendre le point de vue du politique qui raisonne sur des flux et des leviers. C'est une vision très gestionnaire qui s'affranchit totalement de la spécificité et de la nature même du bien 868 . Si nous étions un brin provocateur, on pourrait rappeler que l'un des critères essentiels de I'UNESCO est d'assurer l'authenticité et l'intégrité du bien. Sur la base de ce seul critère, il faudrait protéger les chemins du politique. A contrario, la région Occitanie est engagée auprès de l'Agence des chemins de Compostelle, dont la présidence est assurée par le conseiller régional John Palacin. Cette association loi 1901 a un caractère professionnel, laïc, parapublic et culturel. Elle réunit des collectivités territoriales (régions, départements, communes ou intercommunalités), des hébergeurs, des offices de tourisme, des associations jacquaires ou de valorisation du patrimoine ainsi que des personnes qualifiées. Depuis 2015, dans le cadre d'une mission confiée par l'État, l'Agence anime le réseau des propriétaires, gestionnaires et acteurs du bien culturel « Chemins de Saint-Jacques-de-Compostelle en France » inscrit sur la Liste du patrimoine mondial de l'UNESCO. Elle a également pour rôle de transmettre les valeurs liées à I'héritage culturel des chemins vers Saint-Jacques-de-Compostelle, faire connaître, faire vivre et préserver ce patrimoine pour le transmettre. Cette agence a pleinement conscience de la singularité du bien 868 et de l'enjeu de sa préservation. Elle sait s'entourer de personnalités qualifiées, universitaires et autres acteurs de terrain pour développer et préserver ce patrimoine mais les obstacles propres à sa mission sont considérables. À titre d'exemple, la coordination d'actions de collectivités territoriales de nature différente, avec des champs de compétences qui parfois se juxtaposent nécessite des trésors de diplomatie pour aboutir à une position commune. II en est de même des lourdeurs administratives générées par ces formes nouvelles de collaboration entre différents niveaux de responsabilités et de compétences, convoqués par la spécificité du bien 868. Et lorsque l'on dépasse le champ hexagonal, la collaboration entre la France et l'Espagne sur les chemins de Saint-Jacques-de-Compostelle repose encore trop souvent sur la volonté de quelques pionniers passionnés. Bref, cela semble encore très embryonnaire, malgré le soutien et l'engagement d'acteurs tels que l'Agence des chemins de Compostelle. 


\section{Perceptions et représentations des chemins par les marcheurs-pèlerins}

Rappelons tout d'abord que notre étude est qualitative. Elle repose sur des entretiens approfondis, selon une approche compréhensive menée auprès de 15 marcheurs-pèlerins et complétée par une analyse fine de leurs comptes Instagram, soit un corpus de près de 4000 photos. Le format de cet article ne nous permet pas d'être exhaustif sur cette étude, mais celleci fait ressortir des points saillants en lien direct avec les politiques publiques menées autour de l'objet chemins de Saint-Jacques-de-Compostelle. Premièrement, le chemin est le sujet le plus récurrent dans les entretiens menés et dans l'analyse du corpus de photos publiées sur Instagram. II semble devenir un personnage et cela est d'autant plus vrai que les personnes interrogées pour la plupart nomment le chemin Camino, il s'incarne alors et devient acteur d'un dialogue symbolique par la pensée et le corps du marcheur-pèlerin. Le chemin est aussi présent sur les photos de l'arrivée, que ce soit devant la cathédrale de Compostelle ou devant le cap Finistère. Ce cap Finis Terrae, là où la terre finit, là où le chemin cède la place à l'objectif pour saisir l'immensité de l'océan, vers le couchant. C'est un parallèle qui fait référence à l'eschatologie chrétienne. Deuxièmement, le corollaire du chemin est la marche. Elle est le deuxième sujet le plus traité lors des entretiens et sur les réseaux sociaux observés. Sa large présence est induite par le lien qu'elle représente entre le chemin et le corps du marcheur. Paul, un marcheur-pèlerin interrogé, précise à ce sujet : « Le chemin, on l'a dans les jambes à la fin de la journée ". Cette pratique de la marche renvoie à l'origine anthropologique de l'homme puisque " la marche est l'essence du genre humain » (Picq, 2015, p. 235). La marche engagée sur les chemins de Saint-Jacques est alors sujet. Les photos qui traitent de la marche nous montrent ainsi « qu'il n'est nullement nécessaire d'avoir un but pour marcher... Ce qui importe dans la marche, ce n'est pas le point d'arrivée, mais ce qui se joue en elle, à tout instant " (Le Breton, 2012, p. 30). Troisièmement, toutes les personnes interrogées savent que les chemins sont inscrits sur la liste du patrimoine mondial de l'UNESCO, mais elles ne connaissent pas la composition du bien 868, c'est-à-dire qu'elles ne savent pas que 71 édifices et 7 sections de sentier de grand chemin sont inscrits. Elles citent spontanément quelques ouvrages remarquables qu'elles ont vus, ou visités (I'abbaye de Conques, I'Hôtel-Dieu de Toulouse), mais cela s'est fait naturellement en suivant le chemin. Ces visites ne sont pas la base de leur motivation pour emprunter les chemins. Elles se réjouissent toutes de cette inscription pour des raisons parfois très différentes. Pour Pauline c'est l'assurance de l'entretien et de la conservation des chemins alors que pour Sébastien, c'est enfin une façon de marquer les origines chrétiennes de l'Europe. Toutes sont convaincues que cette visibilité stimule la fréquentation des chemins, permet même à des étrangers issus d'autres cultures de venir sur les chemins. C'est alors que pointe le sujet controversé de l'attraction touristique. Une peur s'exprime, celle de voir les chemins changer de nature, perdre leur caractère religieux ou spirituel, pour devenir un objet de consommation touristique. Paul déclare : " les chemins sont de plus en plus saturés aux beaux jours. Il faut prévoir, anticiper, ce n'est pas ce que je recherche, alors je marche en hiver à présent ». Pour Sébastien, il y a de plus en plus de personnes qui viennent sans nécessairement accepter les contraintes de la marche, il déclare : "est-on vraiment pèlerin lorsque l'on se fait porter son sac ou sa valise d'une étape à une autre avec un chauffeur qui conduit un bus ou une voiture ? À quoi ça rime ? "Sophie nuance le propos en 
précisant : « nous ne faisons pas tous le même chemin, ni pour les mêmes raisons, mais la magie du chemin opère, les rencontres se font ". Voilà une dimension que le développement économique des territoires associés aux chemins de Saint-Jacques ne peut pas occulter: comment associer développement et fréquentation sans dénaturer la composante symbolique des chemins?

La dimension spirituelle est présente chez chacun de nos interviewés. Ils veulent donner du sens à leur marche vers le sanctuaire de Saint-Jacques. Leur motivation n'est pas religieuse pour une large partie de notre corpus, mais la quête de sens, d'engagement et d'introspection est bien présente pour chacun d'entre eux. Le fait de se retrouver avec soi-même sur les chemins et avec d'autres, c'est souvent le fruit d'un passage à l'acte qui se fait le plus souvent à l'issue d'une disruption vécue par la personne interviewée (maladie, décès, changement professionnel, séparation, etc.). Il y a donc un fait spirituel et culturel dans l'engouement vers Saint-Jacquesde-Compostelle avec un cadre religieux souple qui borde les chemins et qui inscrit, accompagne la trajectoire des marcheurs-pèlerins. Ce qui attire, c'est l'imaginaire associé à l'histoire des chemins. Près de $50 \%$ de nos interviewés ne connaissent pas I'histoire de Saint-Jacques-deCompostelle. Certains apprennent à prier en chemin. Ils s'approprient assez souvent une partie des codes et pratiques de la religion catholique pour se construire leur propre spiritualité, il y a une forme de braconnage des usages au sens de Certeau (Certeau, 1990). Cependant, ils adoptent tous Pecten maximus, c'est-à-dire la coquille Saint-Jacques que chaque marcheurpèlerin porte sur son sac à dos ou sur lui-même. C'est un marqueur fort, un signe d'appartenance, à la fois très accessible et distinctif de la communauté des marcheurs-pèlerins. On est assez loin d'une démarche touristique telle que se le représentent certains décideurs politiques locaux. La spiritualité, " bricolée " par cette communauté du « lâcher-prise " (Bertin, 2014) est une clef de compréhension importante de l'engouement et du renouveau de la fréquentation des chemins de Saint-Jacques-de-Compostelle.

En conclusion, les politiques publiques menées sur les chemins de Saint-Jacques-deCompostelle que ce soit par l'UNESCO, le Conseil de l'Europe, le gouvernement français ou les régions, sont caractérisées par une inscription des chemins dans le champ de la culture. Il y a un glissement qui s'opère du but du pèlerinage vers le moyen, à savoir les chemins. Ces politiques s'appuient sur des relais tels que l'Agence des chemins de Compostelle. Le développement économique des zones rurales traversées par les chemins est parfois une injonction. Ce levier de croissance réel ne peut pas se faire sans considérer la réalité historique, spirituelle et religieuse du chemin. La croissance continue de la fréquentation des chemins est une réalité dont il faut se réjouir mais qui nécessite de s'inscrire dans une démarche durable dont la pierre angulaire est de ne pas dénaturer la réalité et la spécificité du bien 868 , inscrit au patrimoine mondial de I'UNESCO. Les politiques doivent penser sur le long terme les chemins et s'engager dans une meilleure coordination des différents acteurs intervenant sur la valorisation et la pérennité des chemins. Développer sans dénaturer, telle est la tension, tel est l'enjeu qui doit guider les acteurs politiques en charge du chemin, c'est le souhait des marcheurs-pèlerins rencontrés dans le cadre de notre étude.

${ }^{1}$ https://oficinadelperegrino.com/en/statistics// (statistiques officielles du bureau des pèlerins de SaintJacques-de-Compostelle) 


\section{Références}

Bertin, G. (2014). La tribu du lâcher prise. Lyon : Éditions du Cosmogone.

Certeau (de), M. (1990). L'Invention du quotidien (vol.1). Paris : Gallimard.

Julia D. (2016). Le voyage aux saints. Les pèlerinages dans l'Occident moderne (XVème-XVIIlème siècle).

Paris : Le Seuil.

Le Breton, D. (2012). Marcher. Eloge des chemins de la lenteur, Paris : Le Seuil.

Malvy, M. (2018, octobre). Communication présentée au Chemin de Saint-Jacques-de-Compostelle en France. Patrimoine, Territoire, Historicité, Toulouse, France.

Picq, P. (2015). La Marche. Sauver le nomade qui est en nous, Paris : Éditions Autrement.

Rucquoi, A. (2014) Mille fois à Compostelle. Pèlerins du Moyen-Age. Paris : Les Belles Lettres.

Vallet, O. (1996). Le routard et la routine. Les cahiers de médiologie, 2, 293-295.

Vielliard, J. (1938). Le Guide du pèlerin de Saint-Jacques-de-Compostelle, texte latin du XIlème siècle, Mâcon : Protat Frères. 